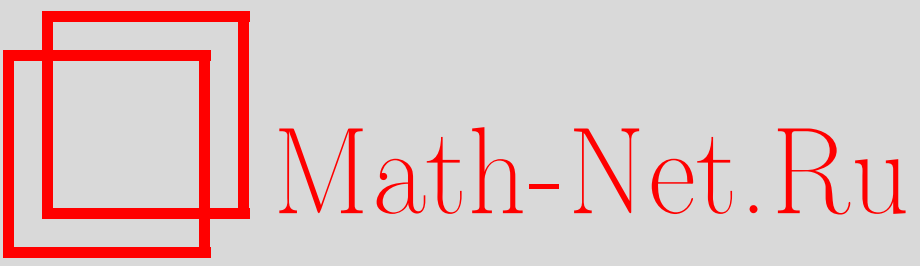

А. А. Солошенко, К. В. Степаньянц, Трехпетлевая $\beta$-функция $N=1$ суперсимметричной электродинамики, регуляризованной высшими производными, ТМФ, 2004, том 140, номер 3, 437-459

DOI: https://doi.org/10.4213/tmf106

Использование Общероссийского математического портала Math-Net.Ru подразумевает, что вы прочитали и согласны с пользовательским соглашением

http://www.mathnet.ru/rus/agreement

Параметры загрузки:

IP: 54.147 .182 .235

26 апреля 2023 г., 16:31:04 
ТЕОРЕТИЧЕСКАЯ

И МАТЕМАТИЧЕСКАЯ

ФИЗИКА

Том 140, № 3

сентябрь, 2004

(C) 2004 г. А. А. Солошенко*, К.В. Степаньянц*

\section{ТРЕХПЕТЛЕВАЯ $\beta$-ФУНКЦИЯ $N=1$ СУПЕРСИММЕТРИЧНОЙ ЭЛЕКТРОДИНАМИКИ, РЕГУЛЯРИЗОВАННОЙ ВЫСШИМИ ПРОИЗВОДНЫМИ}

Вычислен трехпетлевой вклад в эфффективное действие $N=1$ суперсимметричной электродинамики, регуляризованной высшими производными. На основе полученных результатов исследуется проблема аномалий в рассматриваемой модели.

Ключевые слова: суперсимметрия, регуляризация, высшие ковариантные производные, размерная редукция.

\section{1. ВВЕДЕНИЕ}

Хорошо известно [1], что в суперсимметричных теориях аксиальная аномалия и аномалия следа тензора энергии-импульса принадлежат одному супермультиплету. В силу теоремы Адлера-Бардина [2], [3] аксиальная аномалия является чисто однопетлевой, тогда как аномалия следа пропорциональна $\beta$-функции [4]. Поэтому суперсимметрия, по-видимому, должна приводить к тому, что в суперсимметричных теориях поправки к $\beta$-функции должны существовать только в однопетлевом приближении [5]. Этот факт действительно имеет место в $N=2$ суперсимметричных теориях [6]. Однако вычисления, выполненные для $N=1$ суперсимметричных моделей в рамках метода размерной редукции [7], показали, что двухпетлевой вклад в $\beta$-функцию оказывается отличным от нуля. Полученное противоречие в литературе получило название проблемы аномалий.

Предпринимались неоднократные попытки решения этой проблемы. Например, в работе [8] возникновение проблемы аномалий объяснялось различием между вильсоновским эффективным действием и производящим функционалом Г для одночастичнонеприводимых диаграмм. При этом утверждалось, что константа связи в вильсоновском действии имеет только однопетлевую поправку, тогда как константа связи в эффективном действии Г имеет поправки во всех порядках теории возмушений. Различие в поведении констант связи связывалось с некоторым дополнительным аномальным

* Московский государственный университет, Москва, Россия. E-mail: solosh@theor.phys.msu.su; stepan@theor.phys.msu.su 
вкладом. При этом для $\beta$-функции было получено точное во всех порядках выражение, связываюшее ее с аномальной размерностью суперполей материи. Ранее такое выражение было построено в работе [9] исходя из требования ренорминвариантности инстантонных вкладов в эффективное действие и получило название точная $\beta$-функиия Новикова-Шифмана-Вайнштейна-Захарова (НШВЗ). Для случая $N=1$ суперсимметричной электродинамики, которьй будет рассматриваться в этой работе, точная $\beta$-функция НШВЗ имеет следующий вид:

$$
\beta(\alpha)=\frac{\alpha^{2}}{\pi}(1-\gamma(\alpha))
$$

где $\gamma(\alpha)$ - аномальная размерность суперполя материи. Оказалось, что $\beta$-функция НШВЗ согласуется с трехпетлевыми вычислениями, выполненными с использованием размерной редукции в работах [10], только с точностью до переопределения перенормированной константы связи [11]. В принципе схему, в которой получается точная $\beta$-функция, можно построить, устанавливая ее связь с $\overline{\mathrm{MS}}$-схемой в каждом порядке теории возмущений [12]. Кроме того, необходимо заметить, что точная $\beta$-функция НШВЗ также получается не только при использовании размерной редукции, но и в дифференциальной перенормировке [13]. Такое вычисление в двух петлях было проведено в работе [14] для $N=1$ суперсимметричной теории Янга-Миллса.

Еше одним шагом в решении проблемы аномалий является работа [15]. В ней было показано, что преобразования масштабирования полей в суперсимметричных теориях являются аномальными, благодаря чему в зависимости от выбираемой нормировки полей может возникать либо чисто однопетлевой результат, либо точная $\beta$-функция НШВЗ. В случае так называемой голоморфной нормировки, для которой перенормированное действие записывается в виде

$$
\begin{aligned}
S_{\mathrm{ren}}= & \frac{1}{4 e^{2}} Z_{3}\left(e, \frac{\Lambda}{\mu}\right) \operatorname{Re} \int d^{4} x d^{2} \theta W_{a} C^{a b} W_{b}+ \\
& +Z\left(e, \frac{\Lambda}{\mu}\right) \frac{1}{4} \int d^{4} x d^{4} \theta\left(\phi^{*} e^{2 V} \phi+\tilde{\phi}^{*} e^{-2 V} \tilde{\phi}\right),
\end{aligned}
$$

было высказано предположение, что $\beta$-функция является чисто однопетлевой, тогда как при канонической нормировке, в которой

$$
\begin{aligned}
S_{\text {ren }}= & \frac{1}{4 e^{2}} Z_{3}\left(e, \frac{\Lambda}{\mu}\right) \operatorname{Re} \int d^{4} x d^{2} \theta W_{a} C^{a b} W_{b}+ \\
& +\frac{1}{4} \int d^{4} x d^{4} \theta\left(\phi^{*} e^{2 V} \phi+\tilde{\phi}^{*} e^{-2 V} \tilde{\phi}\right),
\end{aligned}
$$

$\beta$-функция имеет поправки от всех порядков теории возмушений и совпадает с точной $\beta$-функцией НШВЗ. Однако такое решение противоречит явным двухпетлевым вычислениям, проведенным в рамках метода размерной редукции.

Было бы естественно предположить, что в голоморфной нормировке чисто однопетлевой результат для $\beta$-функции может возникать при использовании регуляризации 
высшими ковариантными производными [16], дополненной регуляризацией Паули-Вилларса для устранения остаточных однопетлевых расходимостей. Известно, что на однопетлевом уровне результаты вычислений в такой регуляризации всегда согласуются с результатами вычислений в размерной регуляризации (редукции) [17]. Конкретные двухпетлевые вычисления при использовании регуляризации высшими производными были впервые выполнены в работах [18], [19] для $N=1$ суперсимметричной электродинамики. При этом оказалось, что метод высших ковариантных производных позволяет значительно более эффективно проводить исследование квантовых поправок, поскольку он дает возможность правильно вычислять аномальные вклады и лишен противоречий, присуших методу размерной редукции [20]. (Необходимо отметить, что регуляризация с помощью размерной редукции [21], в отличие от размерной регуляризации [22], является математически противоречивой [23].) Более того, метод высших производных позволяет дать простое и естественное решение проблемы аномалий, которое соединяет между собой решения, предложенные в работах [8] и [15]. Детально эти вопросы будут обсуждаться ниже на основе выполненного вычисления трехпетлевой двухточечной функции Грина калибровочного поля.

Статья организована следуюшим образом. Основные сведения об $N=1$ суперсимметричной электродинамике и ее регуляризации с помошью метода высших производных напоминаются в разделе 2. Структура трехпетлевой двухточечной функции Грина калибровочного поля анализируется в разделе 3 , где также показано, что расходимости в этой функции Грина сушествуют только в однопетлевом приближении. В разделе 4 приводится решение проблемы аномалий, следуюшее из результатов данной работы. Полученные результаты обсуждаются в разделе 5 , а некоторые технические детали вычислений приведены в приложениях А и Б.

\section{2. $N=1$ СУПЕРСИММЕТРИЧНАЯ ЭЛЕКТРОДИНАМИКА И РЕГУЛЯРИЗАЦИЯ ВЫСШИМИ ПРОИЗВОДНЫМИ}

$N=1$ суперсимметричная электродинамика в суперпространстве описывается действием

$$
S_{0}=\frac{1}{4 e^{2}} \operatorname{Re} \int d^{4} x d^{2} \theta W_{a} C^{a b} W_{b}+\frac{1}{4} \int d^{4} x d^{4} \theta\left(\phi^{*} e^{2 V} \phi+\tilde{\phi}^{*} e^{-2 V} \tilde{\phi}\right) .
$$

При этом $\phi$ и $\tilde{\phi}$ - киральные суперполя материи, а $V$ - абелево вешественное суперполе, которое в качестве одной из компонент содержит калибровочное поле $A_{\mu}$. Суперполе $W_{a}$ является суперсимметричным аналогом тензора напряженности калибровочного поля и в абелевом случае определяется как

$$
W_{a}=\frac{1}{16} \bar{D}\left(1-\gamma_{5}\right) D\left[\left(1+\gamma_{5}\right) D_{a} V\right]
$$

где суперсимметричная ковариантная производная $D$ записывается в виде

$$
D=\frac{\partial}{\partial \bar{\theta}}-i \gamma^{\mu} \theta \partial_{\mu}
$$


Для того чтобы регуляризовать модель (4) при помоши метода высших ковариантных производных, ее действие модифицируется следуюшим образом:

$$
\begin{aligned}
S_{0} \rightarrow S= & S_{0}+S_{\Lambda}=\frac{1}{4 e^{2}} \operatorname{Re} \int d^{4} x d^{2} \theta W_{a} C^{a b}\left(1+\frac{\partial^{2 n}}{\Lambda^{2 n}}\right) W_{b}+ \\
& +\frac{1}{4} \int d^{4} x d^{4} \theta\left(\phi^{*} e^{2 V} \phi+\tilde{\phi}^{*} e^{-2 V} \tilde{\phi}\right) .
\end{aligned}
$$

Заметим, что в силу абелевости рассматриваемой модели суперполе $W^{a}$ является калибровочно-инвариантным, благодаря чему регуляризуюшее слагаемое будет содержать обычные, а не ковариантные производные.

Квантование модели (6) производится стандартными методами, подробно описанными в книге [24], и здесь не обсуждается. Отметим только, что калибровку удобно фиксировать добавлением слагаемых

$$
S_{\mathrm{gf}}=-\frac{1}{64 e^{2}} \int d^{4} x d^{4} \theta\left(V D^{2} \bar{D}^{2}\left(1+\frac{\partial^{2 n}}{\Lambda^{2 n}}\right) V+V \bar{D}^{2} D^{2}\left(1+\frac{\partial^{2 n}}{\Lambda^{2 n}}\right) V\right),
$$

где

$$
D^{2} \equiv \frac{1}{2} \bar{D}\left(1+\gamma_{5}\right) D, \quad \bar{D}^{2} \equiv \frac{1}{2} \bar{D}\left(1-\gamma_{5}\right) D,
$$

поскольку после добавления таких членов кинетический член калибровочного поля записывается в наиболее простом виде:

$$
S_{\text {gauge }}+S_{\text {gf }}=\frac{1}{4 e^{2}} \int d^{4} x d^{4} \theta V \partial^{2}\left(1+\frac{\partial^{2 n}}{\Lambda^{2 n}}\right) V .
$$

В силу абелевости исследуемой модели диаграммы с духовыми петлями отсутствуют.

Индекс расходимости для модели (6) оказывается равным (см., например, [18])

$$
\omega_{\Lambda}=2-2 n(L-1)-E_{\phi}(n+1),
$$

где $L$ - количество петель, а $E_{\phi}$ - число внешних $\phi$-линий. Поскольку в соответствии с формулой (9) даже при $n \geqslant 2$ в однопетлевых диаграммах по-прежнему сушествуют остаточные расходимости, то необходимо добавить в производящий функционал детерминанты Паули-Вилларса [3]. После этого, принимая во внимание, что в силу суперсимметричной калибровочной инвариантности

$$
V \rightarrow V-\frac{1}{2}\left(A+A^{+}\right), \quad \phi \rightarrow e^{A} \phi, \quad \tilde{\phi} \rightarrow e^{-A} \tilde{\phi}
$$

где $A$-произвольное скалярное киральное суперполе, перенормированное действие рассматриваемой модели может быть представлено в виде

$$
\begin{aligned}
S_{\text {ren }}^{h}= & \frac{1}{4 e^{2}} Z_{3}\left(e, \frac{\Lambda}{\mu}\right) \operatorname{Re} \int d^{4} x d^{2} \theta W_{a} C^{a b}\left(1+\frac{\partial^{2 n}}{\Lambda^{2 n}}\right) W_{b}+ \\
& +Z\left(e, \frac{\Lambda}{\mu}\right) \frac{1}{4} \int d^{4} x d^{4} \theta\left(\phi^{*} e^{2 V} \phi+\tilde{\phi}^{*} e^{-2 V} \tilde{\phi}\right),
\end{aligned}
$$


производящий функционал окажется равным

$$
Z=\int D V D \phi D \tilde{\phi} \prod_{i}\left(\operatorname{det} P V\left(V, M_{i}\right)\right)^{c_{i}} \exp \left(i S_{\text {ren }}^{\mathrm{h}}+i S_{\mathrm{gf}}+i S_{\text {source }}\right)
$$

где

$$
S_{\text {source }}=\int d^{4} x d^{4} \theta J V+\int d^{4} x d^{2} \theta(j \phi+\tilde{j} \tilde{\phi})+\int d^{4} x d^{2} \bar{\theta}\left(j^{*} \phi^{*}+\tilde{j}^{*} \tilde{\phi}^{*}\right)
$$

обозначает все члены с источниками,

$$
\begin{aligned}
& (\operatorname{det} P V(V, M))^{-1}=\int D \Phi D \widetilde{\Phi} \exp \left\{i \left[Z ( e , \frac { \Lambda } { \mu } ) \frac { 1 } { 4 } \int d ^ { 4 } x d ^ { 4 } \theta \left(\Phi^{*} e^{2 V} \Phi+\right.\right.\right. \\
& \left.\left.\left.+\widetilde{\Phi}^{*} e^{-2 V} \widetilde{\Phi}\right)+\frac{1}{2} \int d^{4} x d^{2} \theta M \widetilde{\Phi} \Phi+\frac{1}{2} \int d^{4} x d^{2} \bar{\theta} M \widetilde{\Phi}^{*} \Phi^{*}\right]\right\},
\end{aligned}
$$

а коэффициенты $c_{i}$ удовлетворяют уравнениям

$$
\sum_{i} c_{i}=1, \quad \sum_{i} c_{i} M_{i}^{2}=0 .
$$

Далее мы будем предполагать, что $M_{i}=a_{i} \Lambda$, где $a_{i}$ - некоторые постоянные. Добавление детерминантов Паули-Вилларса позволяет сократить остаточные расходимости во всех однопетлевых диаграммах. Важно отметить, что величина $Z(e, \Lambda / \mu)$ в формулах (10) и (13) является одной и той же. Это необходимо для того, чтобы регуляризовать расходимости в диаграммах с контрчленными вставками, которые не содержат внутренних фотонных линий.

Производящий функционал для связных функций Грина в наших обозначениях записывается в виде

$$
W=-i \ln Z
$$

а эффективное действие получается с помошью преобразования Лежандра:

$$
\Gamma=W-\int d^{4} x d^{4} \theta J V-\int d^{4} x d^{2} \theta(j \phi+\tilde{j} \tilde{\phi})-\int d^{4} x d^{2} \bar{\theta}\left(j^{*} \phi^{*}+\tilde{j}^{*} \tilde{\phi}^{*}\right)
$$

причем источники $J, j$ и $\tilde{j}$ должны быть выражены через поля $V, \phi$ и $\tilde{\phi}$ при помощи уравнений

$$
V=\frac{\delta W}{\delta J}, \quad \phi=\frac{\delta W}{\delta j}, \quad \tilde{\phi}=\frac{\delta W}{\delta \tilde{j}}
$$

Для получения эффективного действия необходимо вычислить все одночастично-неприводимые диаграммы в исследуемом порядке теории возмущений, выражения для которых строятся при помощи правил Фейнмана, которые для рассматриваемой теории могут быть сформулированы следующим образом. 
1. Внешним линиям соответствует множитель

$$
\prod_{E} \int \frac{d^{4} p_{E_{V}}}{(2 \pi)^{4}} V\left(p_{E_{V}}\right) \int \frac{d^{4} p_{E_{\phi}}}{(2 \pi)^{4}} \phi\left(p_{E_{\phi}}\right) \times \cdots \times(2 \pi)^{4} \delta\left(\sum_{E} p_{E}\right),
$$

где индекс $E$ нумерует внешние импульсы.

2. Каждой внутренней линии суперполя $V$ соответствует пропагатор

$$
\frac{8 e^{2}}{\left(k^{2}+i 0\right)\left(1+(-1)^{n} k^{2 n} / \Lambda^{2 n}\right)} \delta^{4}\left(\theta_{1}-\theta_{2}\right)
$$

3. Каждой внутренней линии $\phi-\phi^{*}$ или $\tilde{\phi}-\tilde{\phi}^{*}$ соответствует пропагатор

$$
-\frac{1}{16\left(k^{2}+i 0\right)} \bar{D}^{2} D^{2} \delta^{4}\left(\theta_{1}-\theta_{2}\right)
$$

(Заметим, что действие рассматриваемой теории квадратично по суперполям материи, что позволяет сформулировать более простые правила Фейнмана по сравнению с правилами Фейнмана для модели Весса-Зумино.)

4. Поля Паули-Вилларса присутствуют только в замкнутых петлях. При этом каждой внутренней линии $\Phi-\Phi^{*}$ или $\widetilde{\Phi}-\widetilde{\Phi}^{*}$ соответствует пропагатор

$$
-\frac{1}{16\left(k^{2}-M_{i}^{2}+i 0\right)} \bar{D}^{2} D^{2} \delta^{4}\left(\theta_{1}-\theta_{2}\right)
$$

а каждой внутренней линии $\Phi-\widetilde{\Phi}$ или $\Phi^{*}-\widetilde{\Phi}^{*}-$

$$
\frac{M_{i}}{4\left(k^{2}-M_{i}^{2}+i 0\right)} \bar{D}^{2} \delta^{4}\left(\theta_{1}-\theta_{2}\right) \quad \text { и } \quad \frac{M_{i}}{4\left(k^{2}-M_{i}^{2}+i 0\right)} D^{2} \delta^{4}\left(\theta_{1}-\theta_{2}\right) .
$$

Также для каждой замкнутой петли полей Паули-Вилларса необходимо добавить $-\sum_{i} c_{i}$

5. Каждой замкнутой петле соответствует интегрирование по петлевому импульсу $\int d^{4} k /(2 \pi)^{4}$.

6. Каждая вершина дает интегрирование по соответствуюшему $\theta: \int d^{4} \theta$.

7. Для каждой диаграммы необходимо найти числовой коэффициент, что можно сделать, например, при помощи разложения производящего функционала (11) в ряд по теории возмущений. 
ТРЕХПЕТЛЕВАЯ $\beta$-ФУНКЦИЯ $N=1$ СУПЕРСИММЕТРИЧНОЙ ЭЛЕКТРОДИНАМИКИ 443

\section{3. ТРЕХПЕТЛЕВАЯ ДВУХТОЧЕЧНАЯ ФУНКЦИЯ ГРИНА КАЛИБРОВОЧНОГО ПОЛЯ}

Диаграммы, даюшие вклад в трехпетлевую двухточечную функцию Грина калибровочного поля, показаны на рис. 1-8. При этом по каждой внутренней линии суперполей материи могут распространяться поля $\phi, \tilde{\phi}$ или поля Паули-Вилларса. Поэтому трехпетлевой вклад в эффективное действие можно представить в виде

$$
\begin{aligned}
\Delta \Gamma_{V}^{(3)}= & \operatorname{Re} \int d^{2} \theta \frac{d^{4} p}{(2 \pi)^{4}} W_{a}(p) C^{a b} W_{b}(-p) \times \\
& \times\left(f_{1 \text {-loop }}+f_{\bigcirc}+f_{\bigcirc \mathrm{PV}}+f_{\bigcirc \circ}+f_{\bigcirc_{\mathrm{PV}}}+f_{\bigcirc \bigcirc}+f_{\times \times}+f_{\times}+f_{\times 2}\right),
\end{aligned}
$$

где были использованы следующие обозначения:

$f_{\text {1-loор }}$ - вклад однопетлевых диаграмм, показанных на рис. 1, с учетом вкладов полей Паули-Вилларса;

$f_{\bigcirc}-$ суммарный вклад диаграмм с одной петлей суперполей $\phi$ и $\tilde{\phi}$, показанных на рис. 5;

$f_{\bigcirc_{P V}}-$ вклад диаграмм с одной петлей полей Паули-Вилларса,показанных на рис. 5;

$f_{\bigcirc}$ - вклад двухпетлевых диаграмм, показанных на рис. 2 (без вкладов полей Паули-Вилларса), и трехпетлевых диаграмм, показанных на рис. 4 , которые содержат внутреннюю петлю суперполей материи или однопетлевую контрчленную вставку на внутренней фотонной линии, причем по внешшей петле распространяются поля $\phi$ и $\tilde{\phi}$;

$f_{\bigcirc \circ \mathrm{pV}}-$ вклад двухпетлевых диаграмм, показанных на рис. 2 с внутренней петлей полей Паули-Вилларса, и трехпетлевых диаграмм, показанных на рис. 4, в которых по внешней петле распространяются поля Паули-Вилларса;

$f_{\bigcirc \bigcirc}$ - вклад остальных диаграмм с двумя петлями суперполей материи, показанных на рис. 3 ;

$f_{\times}$- вклад диаграмм со вставкой однопетлевых и двухпетлевых контрчленов, показанных на рис. 6 ;

$f_{\times \times}$- вклад диаграмм с двумя вставками однопетлевых контрчленов, показанных на рис. 8 ;

$f_{\times 2}-$ вклад диаграмм с одной вставкой однопетлевых контрчленов, показанных на рис. 7.

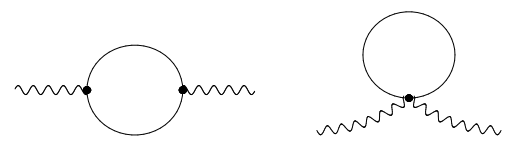

Рис. 1. Однопетлевые диаграммы Фейнмана, дающие нетривиальный вкладв $\beta$-функцию $N=1$ суперсимметричной электродинамики.

Явные выражения, полученные для всех этих величин после вычисления диаграмм Фейнмана, приведены в работе [25]. Поскольку они являются достаточно громоздкими, 


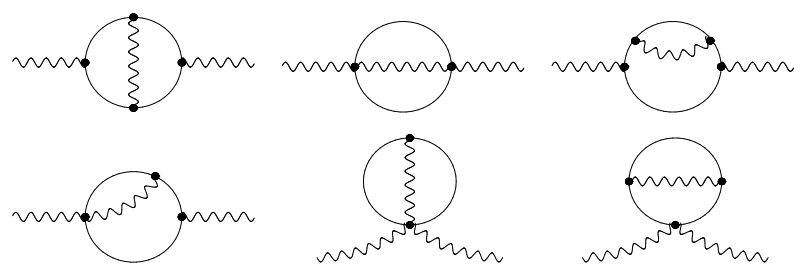

Рис. 2. Двухпетлевые диаграммы Фейнмана, дающие нетривиальный вклад в $\beta$-функцию $N=1$ суперсимметричной электродинамики.

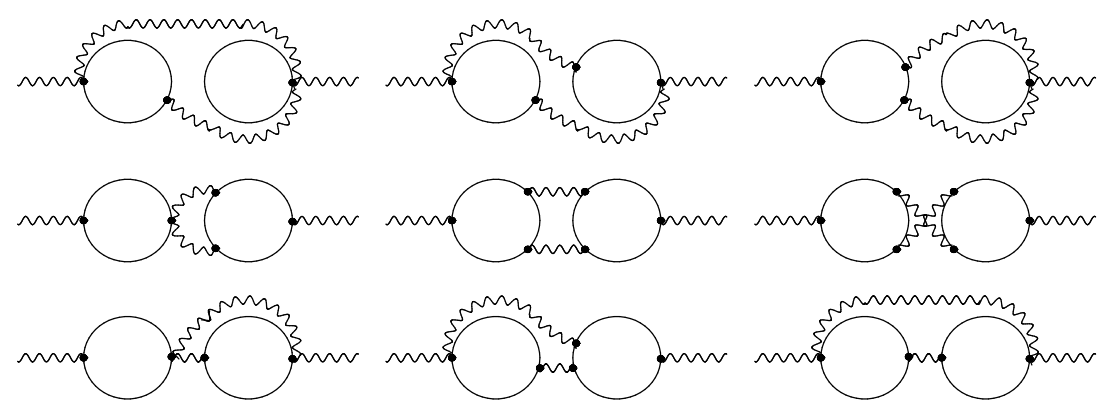

Рис. 3. Диаграммы Фейнмана, имеющие две внутренние петли суперполей материи, к каждой из которых прикреплена своя внешняя линия.

то здесь мы их не выписываем. В качестве примера в приложении А приводится выражение для наиболее нетривиального вклада $f_{\bigcirc}$ и описывается способ вычисления его расходящейся части.

Заметим, что в качестве одной из проверок правильности вычислений использовалось сокращение всех неинвариантных слагаемых, пропорциональных

$$
\int \frac{d^{4} p}{(2 \pi)^{4}} d^{4} \theta V(p, \theta) V(-p, \theta)
$$

Проведем теперь краткий анализ вкладов различных диаграмм.

1. Вклад $f \bigcirc \bigcirc=0$, поскольку замена $\phi \leftrightarrow \tilde{\phi}$ в одной из петель приводит к изменению знака диаграммы. Это означает, что в рассматриваемой теории диаграммы, имеюшие одинаковые суперполя в обоих петлях ( $\phi$ и $\phi$ или $\tilde{\phi}$ и $\tilde{\phi})$, компенсируются диаграммами с петлями разных суперполей $(\phi$ и $\tilde{\phi})$. Для соответствующих вкладов полей Паули-Вилларса ситуация несколько сложнее, но результат оказывается тем же самым.

2. Сумма вкладов $f_{\times \times}, f_{\times}$и $f_{\times 2}$ полностью согласуется с точным выражением для суммы диаграмм с контрчленными вставками

$$
-\ln Z \frac{1}{16 \pi^{2}} \operatorname{Re} \int d^{4} x d^{2} \theta W_{a} C^{a b} W_{b}+\text { конечные члены, }
$$

построенным в работе [20], причем $f_{\times 2}=0$. 

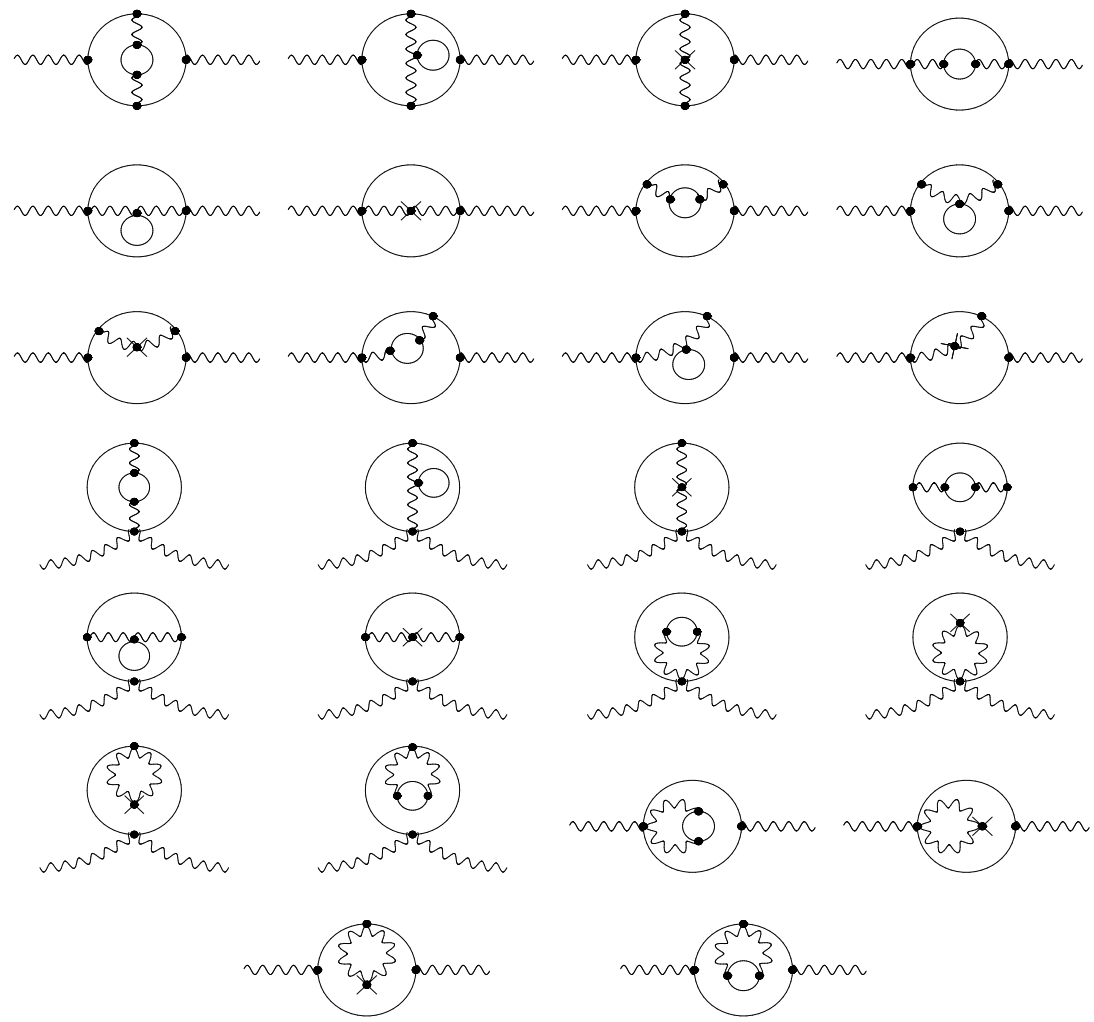

Рис. 4. Диаграммы Фейнмана, дающие нетривиальный вклад в трехпетлевую $\beta$-функцию $N=1$ суперсимметричной электродинамики и соответствующие учету поправок к фотонному пропагатору.

При этом константа $Z$ (см., например, [18]) может быть записана в виде

$$
Z\left(e, \frac{\Lambda}{\mu}\right)=1+\frac{\alpha}{\pi}\left(\ln \frac{\Lambda}{\mu}+g_{1}\right)+\frac{\alpha^{2}}{\pi^{2}}\left(\ln ^{2} \frac{\Lambda}{\mu}+g_{1} \ln \frac{\Lambda}{\mu}\right)-\gamma_{2} \alpha^{2}\left(\ln \frac{\Lambda}{\mu}+g_{2}\right)+O\left(\alpha^{3}\right)
$$

где $\gamma_{2} \alpha^{2}$ представляет собой двухпетлевой вклад в аномальную размерность, а также предполагается, что для компенсации расходимостей в однопетлевом приближении к действию добавлены контрчлены вида

$$
\begin{aligned}
\Delta S= & -\frac{1}{16 \pi^{2}}\left(\ln \frac{\Lambda}{\mu}+b_{1}\right) \operatorname{Re} \int d^{4} x d^{2} \theta W_{a} C^{a b}\left(1+\frac{\partial^{2 n}}{\Lambda^{2 n}}\right) W_{b}+ \\
& +\frac{e^{2}}{16 \pi^{2}}\left(\ln \frac{\Lambda}{\mu}+g_{1}\right) \int d^{4} x d^{4} \theta\left(\phi^{*} e^{2 V} \phi+\tilde{\phi}^{*} e^{-2 V} \tilde{\phi}\right)
\end{aligned}
$$

При этом $b_{1}, g_{1}$ и $g_{2}$ представляют собой произвольные конечные постоянные, выбор которых фиксирует произвол в выборе схемы перенормировки. 

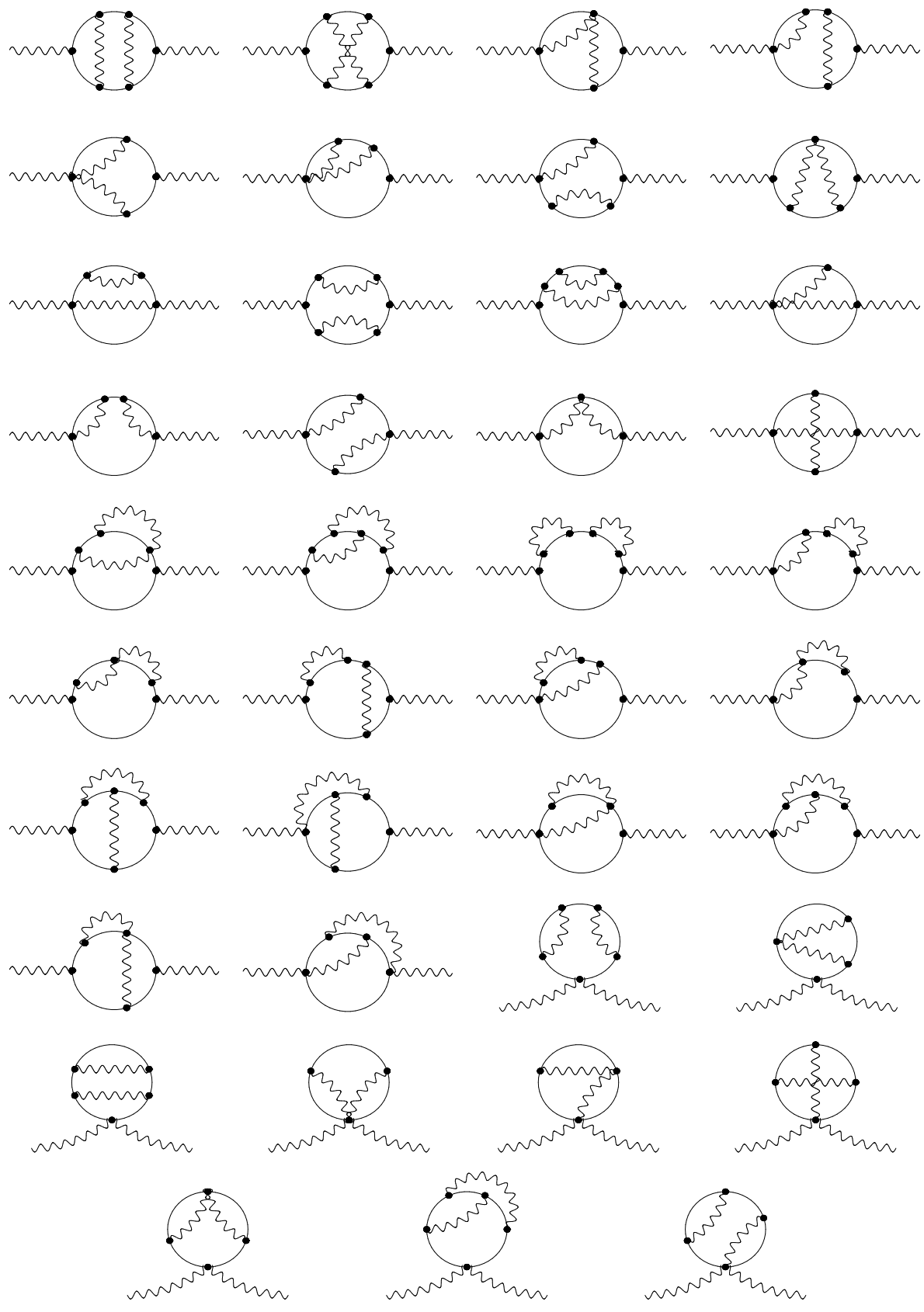

Рис. 5. Диаграммы Фейнмана, дающие нетривиальный вклад в трехпетлевую $\beta$-фуннкцю $N=1$ суперсимметричной электродинамики и имеющие одну внутреннюю петлю суперполей материи.

3. Вклады $f_{\bigcirc_{\mathrm{PV}}}$ и $f_{\bigcirc_{\mathrm{PV}}}$ являются конечными и не влияют на расходящуюся часть эффективного действия. Это может быть доказано следуюшим образом. Интеграл, 

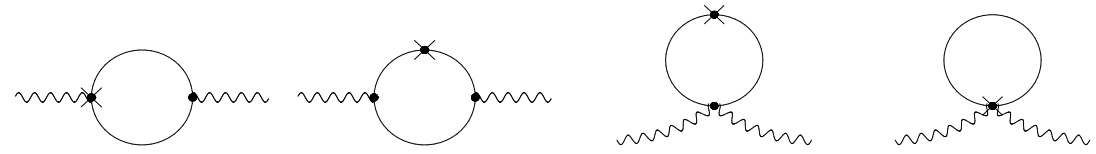

Рис. 6. Диаграммы Фейнмана, дающие нетривиальный вклад в $\beta$-функцию $N=1$ суперсимметричной электродинамики и содержащие вставки однопетлевых и двухпетлевых контрчленов во внутренние линии суперполей материи.
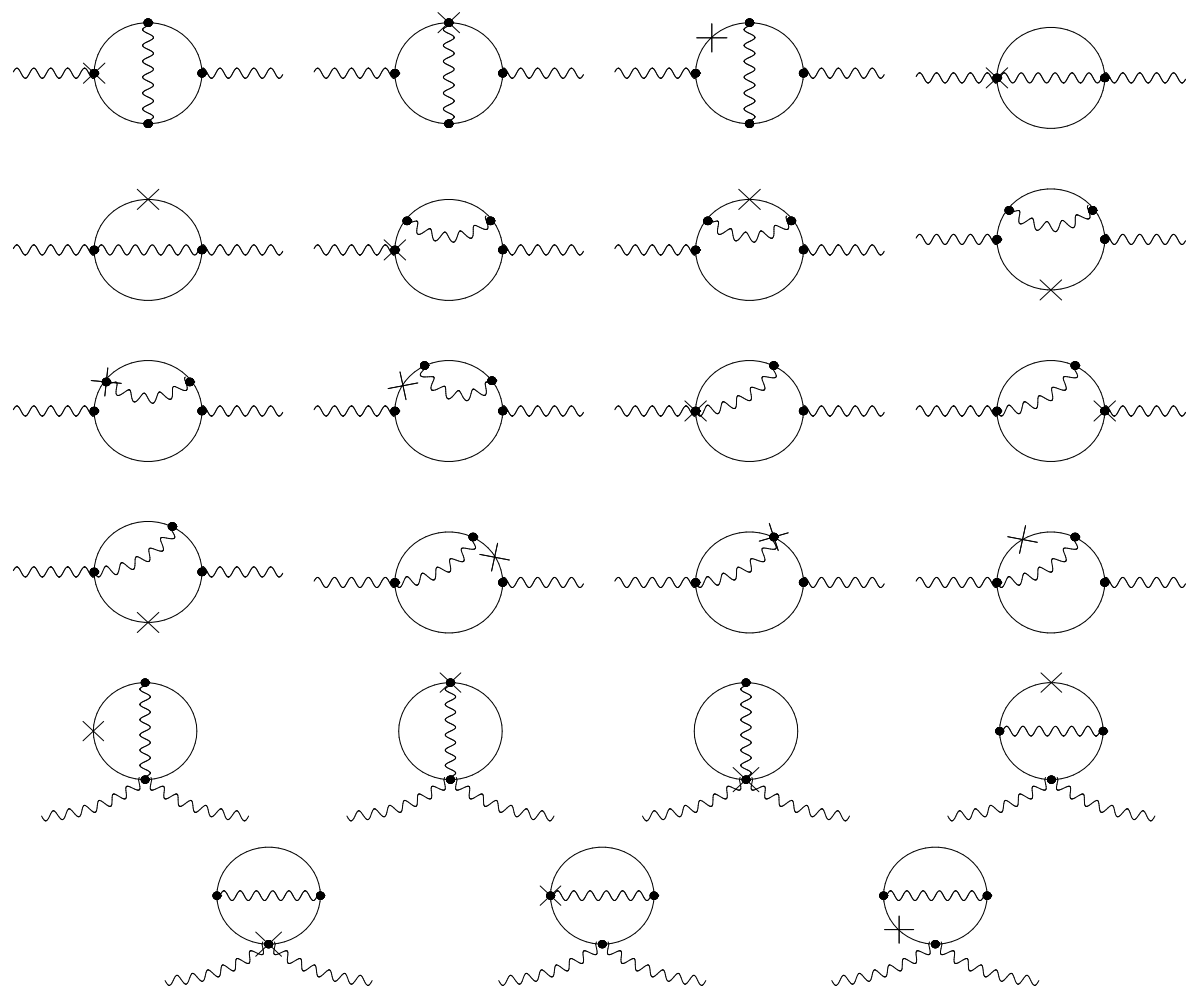

Рис. 7. Диаграммы Фейнмана, дающие нетривиальный вклад в трехпетлевую $\beta$-функцию $N=1$ суперсимметричной электродинамики и содержащие одну вставку однопетлевых контрчленов во внутренние линии суперполей материи.

определяюший вклад $f_{\bigcirc}{ }_{\mathrm{PV}}$, можно представить в виде

$$
f_{\bigcirc_{\mathrm{PV}}}=f_{1}\left(\frac{p}{\Lambda}\right)+\ln \frac{\Lambda}{\mu} f_{2}\left(\frac{p}{\Lambda}\right),
$$

где $f_{1}$ и $f_{2}$ - некоторые функции, аналитические при $p^{2}=0$. При этом если положить $p=0$, то оказывается, что $f_{1}(0)=0$ и $f_{2}(0)=0$, благодаря чему ведушие слагаемые в разложениях этих функций по $p^{2}$ убывают по крайней мере как $p^{2} / \Lambda^{2}$ (детали вычислений можно найти в работе [25]). Это, в свою очередь, означает, что

$$
\lim _{\Lambda \rightarrow \infty} f_{\bigcirc_{\mathrm{PV}}}=0 .
$$




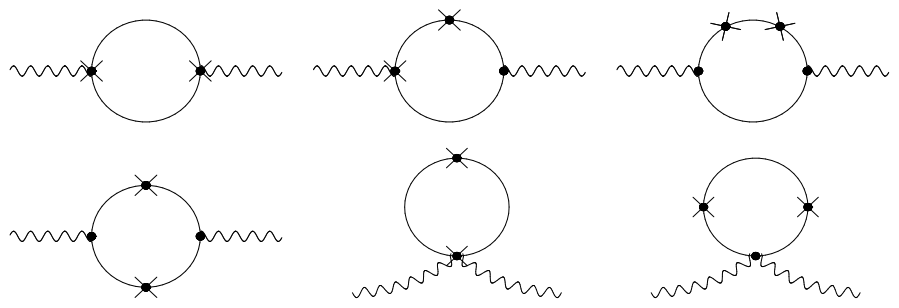

Рис. 8. Диаграммы Фейнмана, дающие нетривиальный вклад в трехпетлевую $\beta$-функцию $N=1$ суперсимметричной электродинамики и содержащие две вставки однопетлевых контрчленов во внутренние линии суперполей материи.

Аналогичным образом доказывается конечность вклада $f_{\bigcirc_{\text {pv }}}$. Действительно, из размерных соображений очевидно, что

$$
f_{\bigcirc_{\mathrm{PV}}}=f\left(\frac{p}{\Lambda}\right)
$$

Однако, полагая $p=0$, можно доказать [25], что $f(0)=0$. Это и означает, что вклад $f_{\bigcirc_{\mathrm{pV}}}$ является конечным и исчезает при снятии регуляризации.

Конечность величин $f_{\bigcirc_{\mathrm{PV}}}$ и $f_{\bigcirc_{\mathrm{PV}}}$ означает, что сумма всех диаграмм с внутренними петлями полей Паули-Вилларса, имеющих внутренние фотонные линии, является конечной. При этом расходимости, вообще говоря, могут сушествовать в отдельных таких диаграммах. Однако поскольку регуляризация Паули-Вилларса по своему определению подразумевает сушествование расходимостей в отдельных диаграммах и их сокрашение при суммировании диаграмм, то дополнительная регуляризация в данном случае не требуется.

4. Анализ оставшихся выражений $f_{\bigcirc}$ и $f_{\bigcirc}$ является достаточно нетривиальным, поскольку содержашиеся в них интегралы имеют достаточно сложную структуру. Однако достаточно очевидно, что эти интегралы являются функциями переменных $e_{0}, \Lambda / p$ (в рассматриваемых вкладах учитываются все контрчленные вставки на линиях калибровочного поля, благодаря чему зависимость от $e$ и $\Lambda / \mu$ объединяется в зависимость от $\left.e_{0}=e_{0}(e, \Lambda / \mu)\right)$.

В работе [25] показано, что для вкладов $f_{\bigcirc}$ и $f_{\bigcirc}$ сушествует предел

$$
\left.\Lambda \frac{d}{d \Lambda} f\left(e_{0}, \frac{\Lambda}{p}\right)\right|_{p=0}
$$

(для вклада $f_{\bigcirc}$ это доказано в приложении $\mathrm{A}$ ), причем с точностью до членов рассматриваемого порядка в разложении по константе связи этот предел может быть записан в виде

$$
\left.\frac{1}{16 \pi^{2}} \Lambda \frac{d}{d \Lambda}\left(z_{1}+z_{2}+\frac{1}{2} z_{1}^{2}\right)\right|_{p=0},
$$


где через $z_{1}$ и $z_{2}$ обозначены следующие евклидовы интегралы:

$$
\begin{aligned}
z_{1} \equiv & \int \frac{d^{4} k}{(2 \pi)^{4}} \frac{2 e^{2}}{k^{2}(k+p)^{2}\left(1+k^{2 n} / \Lambda^{2 n}\right)}, \\
z_{2} \equiv & -\int \frac{d^{4} k}{(2 \pi)^{4}} \frac{d^{4} l}{(2 \pi)^{4}} \frac{4 e^{4}}{k^{2} l^{2}(k+p)^{2}(l+p)^{2}\left(1+k^{2 n} / \Lambda^{2 n}\right)\left(1+l^{2 n} / \Lambda^{2 n}\right)}- \\
& -\int \frac{d^{4} k}{(2 \pi)^{4}} \frac{d^{4} l}{(2 \pi)^{4}} \frac{4 e^{4}}{k^{2} l^{2}(l+p)^{2}(k+l+p)^{2}\left(1+k^{2 n} / \Lambda^{2 n}\right)\left(1+l^{2 n} / \Lambda^{2 n}\right)}+ \\
& +\int \frac{d^{4} k}{(2 \pi)^{4}} \frac{d^{4} l}{(2 \pi)^{4}} \frac{4 e^{4}(k+l+2 p)^{2}}{k^{2}(k+p)^{2} l^{2}(l+p)^{2}(k+l+p)^{2}\left(1+k^{2 n} / \Lambda^{2 n}\right)\left(1+l^{2 n} / \Lambda^{2 n}\right)}- \\
& -\int \frac{d^{4} k}{(2 \pi)^{4}} \frac{4 e^{4}}{k^{2}(k+p)^{2}\left(1+k^{2 n} / \Lambda^{2 n}\right)^{2}}\left(\int \frac{d^{4} l}{(2 \pi)^{4}} \frac{1}{l^{2}(k+l)^{2}}-\right. \\
& \left.-\sum_{i} c_{i} \int \frac{d^{4} l}{(2 \pi)^{4}} \frac{1}{\left(l^{2}+M_{i}^{2}\right)\left((k+l)^{2}+M_{i}^{2}\right)}-\frac{1}{8 \pi^{2}}\left(\ln \frac{\Lambda}{\mu}+b_{1}\right)\left(1+\frac{k^{2 n}}{\Lambda^{2 n}}\right)\right) .
\end{aligned}
$$

Учитывая сушествование предела (24), можно записать

$$
f_{\bigcirc}+f_{\bigcirc \circ}=\frac{1}{16 \pi^{2}}\left(z_{1}+z_{2}+\frac{1}{2} z_{1}^{2}\right)+\text { конечные члены. }
$$

(Несложно убедиться, что с точностью до членов рассматриваемого порядка в разложении по константе связи величина $z_{1}+z_{2}+z_{1}^{2} / 2$ также зависит только от $e_{0}$ и $\Lambda$.)

Обратим теперь внимание на то, что величины $z_{1}$ и $z_{2}$ присутствуют в выражении для двухпетлевой двухточечной функции Грина суперполя материи. Более точно, в соответствии с результатами работы [19] члены эффективного действия, квадратичные по полям материи и не содержашие поля $V$, с точностью до членов рассматриваемого порядка малости можно записать в виде

$$
\Gamma_{\phi}^{(2)}=\frac{1}{4} \int d^{4} \theta \frac{d^{4} p}{(2 \pi)^{4}}\left(\phi^{*}(p, \theta) \phi(-p, \theta)+\tilde{\phi}^{*}(p, \theta) \tilde{\phi}(-p, \theta)\right) Z G,
$$

где

$$
G=1-z_{1}-z_{2}+O\left(\alpha^{3}\right)
$$

представляет собой результат вычисления двухпетлевых диаграмм, показанных на рис. 9, без учета контрчленных вставок на линиях суперполей материи, а константа перенормировки $Z$ дается формулой (18). При этом важно отметить, что величина $Z G$ по построению является конечной с точностью до членов порядка $\alpha^{3}$.

Собирая все вклады, получаем, что сумма диаграмм, определяюших двух- и трехпетлевой вклады в двухточечную функцию калибровочного поля в $N=1$ суперсимметричной электродинамике, с точностью до членов порядка $\alpha^{3}$ может быть записана в виде

$$
\begin{aligned}
\Delta \Gamma_{V}^{(2,3)}= & \frac{1}{16 \pi^{2}} \operatorname{Re} \int d^{2} \theta \frac{d^{4} p}{(2 \pi)^{4}} W_{a}(p) C^{a b} W_{b}(-p) \times \\
& \times\left(z_{1}+z_{2}+\frac{1}{2} z_{1}^{2}-\ln Z+\text { конечные члены }\right)= \\
= & -\frac{1}{16 \pi^{2}} \operatorname{Re} \int d^{2} \theta \frac{d^{4} p}{(2 \pi)^{4}} W_{a}(p) C^{a b} W_{b}(-p)(\ln (Z G)+\text { конечные члены }) .
\end{aligned}
$$

4 Теоретическая и математическая физика, т. 140, № 3, 2004 г. 

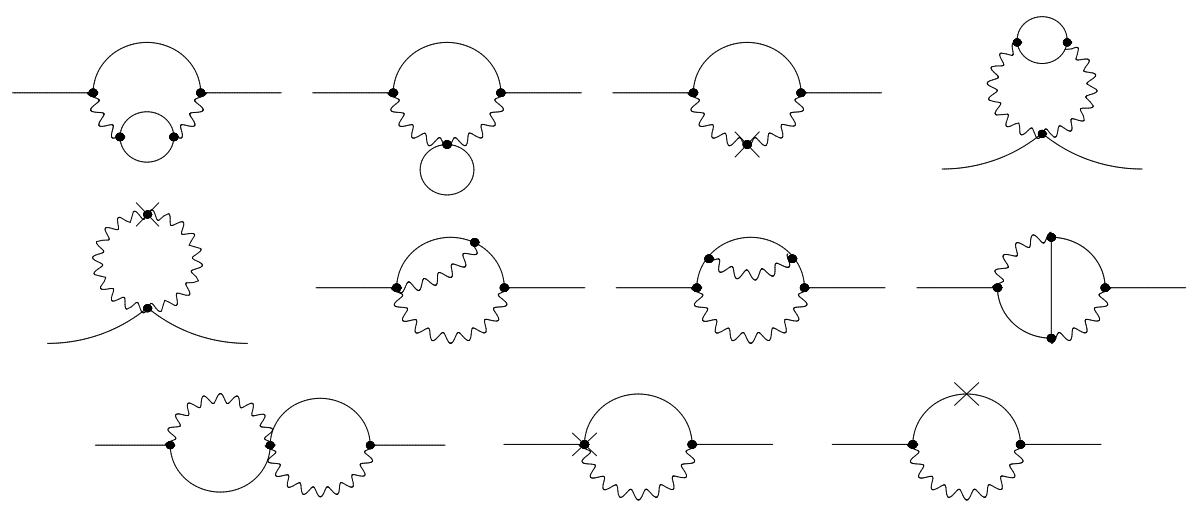

Рис. 9. Диаграммы Фейнмана, дающие нетривиальный вклад в двухпетлевую аномальную размерность $N=1$ суперсимметричной электродинамики.

При этом видно, что двух- и трехпетлевой вклады автоматически оказьваются конечными и контрчлены необходимо добавлять только в однопетлевом приближении. Поэтому при использовании регуляризации высшими производными будет справедливо равенство

$$
\frac{4 \pi^{2}}{e_{0}^{2}}=\frac{\pi}{\alpha}-\ln \frac{\Lambda}{\mu}-b_{1}+O\left(\alpha^{3}\right)
$$

которое свидетельствует о том, что расходимости в двухточечной функции Грина калибровочного поля присутствуют только в однопетлевом приближении.

Окончательный результат для вклада двухточечной функции Грина калибровочного поля в перенормированное эффективное действие в трехпетлевом приближении запишется в виде

$$
\Delta \Gamma_{V}=\frac{1}{16 \pi^{2}} \operatorname{Re} \int d^{2} \theta \frac{d^{4} p}{(2 \pi)^{4}} W_{a}(p) C^{a b} W_{b}(-p)\left(\ln \frac{\mu}{p}-\ln (Z G)+\text { конечные члены }\right)
$$

и имеет поправки от всех порядков теории возмушений.

\section{4. РЕШЕНИЕ ПРОБЛЕМЫ АНОМАЛИЙ}

В рассматриваемой теории аксиальный ток $j_{5}^{\mu}$, суперток $S^{\mu}$ и тензор энергииимпульса $\Theta^{\mu \nu}$ являются компонентами одного вешественного векторного суперполя

$$
J^{\mu}=j_{5}^{\mu}+\bar{\theta} S^{\mu}+\bar{\theta} \gamma_{\nu} \gamma_{5} \theta \Theta^{\mu \nu}+\cdots,
$$

которое в терминах суперполей $V, W_{a}, \phi$ и $\tilde{\phi}$ может быть записано в виде

$$
\begin{aligned}
J^{\mu}= & \frac{1}{e_{0}^{2}} W^{*} \gamma^{\mu} W-\frac{1}{12} Z\left(3 \bar{D}_{a}\left(\phi^{*} e^{2 V}\right) e^{-2 V}\left(\gamma^{\mu}\left(1+\gamma_{5}\right) D\right)_{a}\left(e^{2 V} \phi\right)+\right. \\
& \left.+3 \bar{D}_{a}\left(\tilde{\phi}^{*} e^{-2 V}\right) e^{2 V}\left(\gamma^{\mu}\left(1+\gamma_{5}\right) D\right)_{a}\left(e^{-2 V} \tilde{\phi}\right)-2 \bar{D} \gamma^{\mu} \gamma_{5} D\left(\phi^{*} e^{2 V} \phi+\tilde{\phi}^{*} e^{-2 V} \tilde{\phi}\right)\right) .
\end{aligned}
$$


Формулы (30) и (31) определяют выражения для $j_{5}^{\mu}, S^{\mu}$ и $\Theta^{\mu \nu}$, которые в явном виде могут быть получены с использованием компонентных разложений суперполей теории.

Используя выражение для дивергенции аксиального тока, несложно проверить, что на квантовом уровне закон сохранения тока $J^{\mu}$ должен выглядеть следующим образом:

$$
\left\langle\left(1+\gamma_{5}\right) \gamma^{\mu} D_{c} J_{\mu}\right\rangle=\frac{1}{8 \pi^{2}}\left(1+\gamma_{5}\right) D_{c}\left(W_{a} C^{a b} W_{b}\right)
$$

При этом угловые скобки обозначают среднее, которое соответствует функционалу (11):

$$
\begin{aligned}
\langle A\rangle \equiv & \frac{1}{Z[j=0, J]} \int D V D \phi D \tilde{\phi} A[\phi, \tilde{\phi}, V] \prod_{i}\left(\operatorname{det} P V\left(V, M_{i}\right)\right)^{c_{i}} \times \\
& \times \exp \left(i S_{\text {ren }}^{\mathrm{h}}+i S_{\mathrm{gf}}+i S_{\text {source }}\right)
\end{aligned}
$$

где источник $J$ необходимо выразить через поля с помощью (15).

Однако, с другой стороны, можно проверить, что аномалия следа тензора энергииимпульса $\Theta_{\mu \nu}$ должна быть пропорциональна $\beta$-функции, определяемой как

$$
\beta=\frac{d}{d \ln \mu}\left(\frac{e^{2}}{4 \pi}\right)
$$

где зависимость перенормированной константы связи $е$ от затравочной константы связи $e_{0}$ и $\Lambda / \mu$ определяется из уравнения

$$
\frac{1}{e^{2}} Z_{3}\left(e, \frac{\Lambda}{\mu}\right)=\frac{1}{e_{0}^{2}}
$$

Более точно, имеет место равенство

$$
\left\langle\left(1+\gamma_{5}\right) \gamma^{\mu} D_{c} J_{\mu}\right\rangle=\frac{\beta\left(\alpha_{0}\right)}{\alpha_{0}^{2}} \frac{1}{8 \pi}\left(1+\gamma_{5}\right) D_{c}\left(W_{a} C^{a b} W_{b}\right) .
$$

Поэтому суперсимметрия требует, чтобы $\beta$-функция (32) полностью определялась однопетлевым приближением:

$$
\beta(\alpha)=\frac{\alpha^{2}}{\pi}
$$

Именно это равенство получается при дифференцировании формулы $(28)$ по $\ln \mu$. Поэтому полученные результаты полностью согласуются со структурой супермультиплета аномалий.

Отметим, что можно определять $\beta$-функцию другим способом. Действительно, рассмотрим поперечную часть двухточечной функции Грина калибровочного поля:

$$
\begin{aligned}
& \left.\Pi_{1 / 2} \int d^{4} x d^{4} y \frac{\delta^{2} \Gamma}{\delta V_{x} \delta V_{y}}\right|_{J=0} e^{i p_{\mu} x^{\mu}+i q_{\mu} y^{\mu}}= \\
& =\frac{1}{8 \pi}(2 \pi)^{4} \delta^{4}(p+q) p^{2} \Pi_{1 / 2} \delta^{4}\left(\theta_{x}-\theta_{y}\right) d^{-1}\left(\alpha, \frac{\mu}{p}\right),
\end{aligned}
$$


где

$$
\Pi_{1 / 2} \equiv-\frac{1}{16 \partial^{2}} D^{a} \bar{D}^{2} C_{a b} D^{b}
$$

Тогда соотношение

$$
\tilde{\beta}(d(\alpha, x)) \equiv-\left.\frac{\partial}{\partial \ln x} d(\alpha, x)\right|_{x=1}
$$

будет определять $\beta$-функцию Гелл-Манна-Лоу.

Дифференцируя формулу (29) по $\ln p$, мы получаем, что

$$
\tilde{\beta}(\alpha)=\frac{\alpha^{2}}{\pi}(1-\tilde{\gamma}(\alpha))
$$

где

$$
\tilde{\gamma} \equiv \frac{\partial}{\partial \ln x} \ln (Z G), \quad x \equiv \frac{\mu}{p},
$$

и было принято во внимание, что произведение $Z G$ зависит от $\alpha$ и $\mu / p$. При этом видно, что величина $\tilde{\beta}$ имеет поправки во всех порядках теории возмущений.

Таким образом, $\beta$-функция, которая пропорциональна аномалии следа, является чисто однопетлевой, тогда как физическая $\beta$-функция, связанная с перенормированным эффективным действием, имеет поправки во всех порядках теории возмушений. Это не противоречит ни аргументам, основанным на структуре супермультиплета аномалий, ни петлевым вычислениям. Остается только ответить на вопрос о том, почему $\beta$-функции, определенные различными способами, оказываются различными.

Обычно в теории поля оба этих определения считаются эквивалентными с точностью до произвола в определении перенормированной константы связи. Однако это имеет место только в том случае, когда производящий функционал не зависит от точки нормировки $\mu$. Действительно, если эффективное действие не зависит от точки нормировки $\mu$ при фиксированном значении $e_{0}$, то дифференцируя уравнение $(33)$ по $\ln \mu$, мы получим

$$
0=\tilde{\beta}(d(\alpha, x))-\beta(\alpha) \frac{\partial}{\partial \alpha} d(\alpha, x) \text {. }
$$

В частности, при $x=1$ справедливо равенство

$$
\tilde{\beta}(\tilde{\alpha})=\beta(\alpha) \frac{d \tilde{\alpha}}{d \alpha},
$$

где $\tilde{\alpha} \equiv d(\alpha, 1)$. Таким образом, если производящий функционал не зависит от $\mu$, то приведенные выше определения $\beta$-функции эквивалентны.

Однако в рассматриваемом случае производящий функционал (11) на самом деле зависит от точки нормировки $\mu$ при фиксированном значении $e_{0}$. Действительно, зависимость от $\mu$ в формуле (11) возникает из-за зависяшей от $\mu$ константы перенормировки $Z$. Эту зависимость можно было бы попытаться исключить при помощи замены $\phi \rightarrow Z^{-1 / 2} \phi$. Однако в соответствии с результатами работы [15] такая замена имеет аномальный якобиан, равный экспоненте от величины (17) (этот якобиан легко вычисляется в методе высших производных и фактически представляет собой экспоненту от 
суммы диаграмм с контрчленными вставками). Поскольку выражение (17) содержит $\ln Z$, который зависит от $\mu$, то даже после замены $\phi \rightarrow Z^{-1 / 2} \phi$ производяший функционал (11) будет содержать $\ln Z$ и, следовательно, зависеть от $\mu$. Поэтому уравнение (34), которое связывало бы $\beta$-функции, определенные различными способами, теперь не имеет места. Поэтому функции $\beta$ и $\tilde{\beta}$ в рассматриваемом случае не являются эквивалентными.

В принципе можно определять производящий функционал так, чтобы он не зависел от точки нормировки. Этого можно добиться двумя способами. Мы можем предположить, что затравочная константа связи $e_{0}$ не фиксирована, а некоторым специальным образом зависит от $\mu$. В этом случае $\beta$-функция (32) будет содержать поправки от всех порядков теории возмушений, но уже не будет пропорциональна аномалии следа. Другая возможность заключается в том, чтобы использовать каноническую нормировку суперполей материи и определять производящий функционал как

$$
Z=\int D V D \phi D \tilde{\phi} \prod_{i}\left(\operatorname{det}^{\prime} P V\left(V, M_{i}\right)\right)^{c_{i}} \exp \left(i S_{\text {ren }}^{\mathrm{c}}+i S_{\mathrm{gf}}+i S_{\text {source }}\right)
$$

где

$$
\begin{aligned}
S_{\text {ren }}^{\text {c }}= & \frac{1}{4 e^{2}} Z_{3}\left(e, \frac{\Lambda}{\mu}\right) \operatorname{Re} \int d^{4} x d^{2} \theta W_{a} C^{a b}\left(1+\frac{\partial^{2 n}}{\Lambda^{2 n}}\right) W_{b}+ \\
& +\frac{1}{4} \int d^{4} x d^{4} \theta\left(\phi^{*} e^{2 V} \phi+\tilde{\phi}^{*} e^{-2 V} \tilde{\phi}\right),
\end{aligned}
$$

а детерминанты Паули-Вилларса определяются как

$$
\begin{array}{r}
\left(\operatorname{det}^{\prime} P V(V, M)\right)^{-1} \equiv \int D \Phi D \widetilde{\Phi} \exp \left\{i \left[\frac { 1 } { 4 } \int d ^ { 4 } x d ^ { 4 } \theta \left(\Phi^{*} e^{2 V} \Phi+\right.\right.\right. \\
\left.\left.\left.+\widetilde{\Phi}^{*} e^{-2 V} \widetilde{\Phi}\right)+\frac{1}{2} \int d^{4} x d^{2} \theta M \widetilde{\Phi} \Phi+\frac{1}{2} \int d^{4} x d^{2} \bar{\theta} M \widetilde{\Phi}^{*} \Phi^{*}\right]\right\}
\end{array}
$$

(Вновь кинетический член полей Паули-Вилларса выбирается так, чтобы все расходимости теории были регуляризованы.)

Тогда $\beta$-функция (32) будет пропорциональна аномалии следа, но в этом случае не справедлива теорема Адлера-Бардина. Таким образом, в обоих случаях выполнение равенства (34) не противоречит структуре супермультиплета аномалий.

Важно заметить, что для всех определений производящего функционала перенормированное эффективное действие в физической схеме перенормировки и функция Гелл-Манна-Лоу будут одни и те же.

\section{5. ЗАКЛЮЧЕНИЕ}

В данной работе было проведено вычисление трехпетлевой двухточечной функции Грина калибровочного поля $N=1$ суперсимметричной электродинамики при использовании регуляризации высшими производными. Было установлено, что при стандарт- 
ном определении производящего функционала расходимости в этой функции Грина присутствуют только в однопетлевом приближении, однако конечная часть содержит вклады от всех порядков теории возмущений и связана с двухточечной функцией Грина суперполя материи. При этом доказано, что отсутствуют какие-либо противоречия со структурой супермультиплета аномалий.

Полученные результаты полностью подтверждают результаты работы [20], в которой было указано, что как отличие результата для $\beta$-функции $(32)$, полученного с использованием высших производных, от результата, полученного с помощью размерной редукции, так и сама проблема аномалий связаны с математической противоречивостью метода размерной редукции. В частности, при регуляризации высшими производными теорема Адлера-Бардина справедлива для схем перенормировки, не нарушающих суперсимметрию, что, по всей видимости, не выполняется при регуляризации методом размерной редукции [26].

Подчеркнем, что результаты данной работы согласуются с решением проблемы аномалий, предложенным в [8]. Основное различие при этом заключается в том, что в соответствии с нашими результатами однопетлевым является перенормированное действие $S_{\text {ren }}$, тогда как в [8] предполагалось, что однопетлевым является вильсоновское действие $S_{\mathrm{W}}$. Поэтому с точностью до замены $S_{\mathrm{W}} \rightarrow S_{\mathrm{ren}}$ (которая придает осмысленность формуле $\left\langle e^{i S_{\mathrm{W}}}\right\rangle=e^{i \Gamma}$, сушественно используемой в [8]) наши результаты согласуются с решением проблемы аномалий, предложенным Шифманом и Вайнштейном. Также полученные результаты согласуются и с выводами, сделанными в работе [15].

$\mathrm{K}$ сожалению, проведенное исследование пока ограничено абелевым случаем. Для суперсимметричной теории Янга-Миллса вычисления с использованием метода высших ковариантных производных представляют большую сложность, поскольку введение в теорию слагаемого с высшими ковариантнылми производными [27] существенно усложняет форму действия. В этом случае значительно упростить ситуацию может замена высших ковариантных производных на обычные производные. При этом, конечно, будет нарушена калибровочная инвариантность, что может привести к нарушению тождеств Уорда некоторыми локальными неинвариантными слагаемыми. Тем не менее выбор специальной схемы вычитаний позволяет восстановить калибровочную инвариантность и использовать при вычислениях неинвариантные регуляризации [28]. Для абелевых суперсимметричных теорий такая схема построена в работе [29], а для неабелевых - в [30]. Поэтому вполне возможно, что применение указанной выше регуляризации к суперсимметричным теориям Янга-Миллса позволит провести аналогичные вычисления и в неабелевом случае. В настоящий момент эта работа находится в процессе выполнения.

\section{Пример вычисления расходящейся части интеграла, регуляризованного высшими производными}

В качестве примера, иллюстрируюшего вычисление трехпетлевых вкладов, рассмот- 
рим выражение

$$
\begin{aligned}
f_{\bigcirc}= & -i \int \frac{d^{4} k}{(2 \pi)^{4}} \frac{d^{4} l}{(2 \pi)^{4}} \frac{d^{4} q}{(2 \pi)^{4}} \times \\
& \times \frac{e^{4}}{k^{2}\left(1+(-1)^{n} k^{2 n} / \Lambda^{2 n}\right) l^{2}\left(1+(-1)^{n} l^{2 n} / \Lambda^{2 n}\right) q^{2}(q+p)^{2}(q+k+l)^{2}} \times \\
& \times\left\{\frac{2 k^{2}\left((2 q+k+l)^{2}+(2 q+k+l+p)^{2}-p^{2}\right)}{(q+k)^{2}(q+l)^{2}(q+k+p)^{2}}-\frac{4 p^{2}}{(q+k)^{2}(q+k+p)^{2}}-\right. \\
& -\frac{4(q+p)^{2}}{(q+k)^{2}(q+k+p)^{2}}+\frac{4)^{2}}{(q+k+p)^{2}(q+k+l+p)^{2}}+\frac{4 k^{2}}{(k+q)^{2}}- \\
& -\frac{2 p^{4}}{(q+k)^{2}(q+k+p)^{2}(q+k+l+p)^{2}}-\frac{2(k+l)^{2}}{(q+k)^{2}}- \\
& -\frac{2 k^{2} p^{2}}{(q+k)^{2}(q+k+p)^{2}(q+k+l+p)^{2}}-\frac{4(2 q+k+l+p)^{2}}{(q+k+l+p)^{2}(q+k)^{2}}+ \\
& +\frac{2(k+l)^{2} p^{2}}{(q+k)^{2}(q+k+p)^{2}(q+k+l+p)^{2}}-\frac{2 q+k+k)^{2}(q+l)^{2}}{(q+k+l+p)^{2}(q+l)^{2}(q+k+p)^{2}}+ \\
& \left.+\frac{2 p^{2}(2 q+k+l+p)^{2}}{(q+k+l+p)^{2}(q+l)^{2}(q+k+p)^{2}}\right\} .
\end{aligned}
$$

Для того чтобы вычислить его расходящуюся часть, мы докажем, что существует предел

$$
\left.\Lambda \frac{d}{d \Lambda} f_{\bigcirc}\right|_{p=0} .
$$

После несложных преобразований выражение (39) при $p=0$ в пространстве Евклида может быть представлено в виде

$$
\begin{aligned}
\left.\Lambda \frac{d}{d \Lambda} f_{\bigcirc}\right|_{p=0}= & \Lambda \frac{d}{d \Lambda} \int \frac{d^{4} k}{(2 \pi)^{4}} \frac{d^{4} l}{(2 \pi)^{4}} \frac{d^{4} q}{(2 \pi)^{4}} \frac{2 e^{4}}{k^{2}\left(1+k^{2 n} / \Lambda^{2 n}\right) l^{2}\left(1+l^{2 n} / \Lambda^{2 n}\right)} \times \\
& \times \frac{1}{q^{4}(q+l)^{2}}\left\{\frac{2}{(q+k)^{2}}-\frac{2 k^{2}}{(q+k)^{4}}-\right. \\
& -\frac{12 k^{2}}{(q+k)^{2}(q+k+l)^{2}}+\frac{4(q+l)^{2}}{(q+k)^{2}(q+k+l)^{2}}+ \\
& \left.+\frac{2\left(k^{4}+k^{2} l^{2}\right)}{(q+k)^{4}(q+k+l)^{2}}+\frac{2 k^{2}(k+l)^{2}}{(q+k)^{2}(q+k+l)^{4}}-\frac{(k+l)^{2}}{(q+k+l)^{4}}\right\} .
\end{aligned}
$$

Заметим, что, несмотря на кажущееся существование инфракрасных расходимостей в этом выражении, оно, как будет показано ниже, является конечным. Поэтому в принципе всегда можно считать, что из области интегрирования в этом выражении (а также в других аналогичных выражениях) исключены бесконечно малые окрестности вблизи сингулярных точек. 
Для упрощения формулы (40) используются некоторые тождества, например

$$
\begin{aligned}
I & \equiv \int \frac{d^{4} k}{(2 \pi)^{4}} \frac{d^{4} l}{(2 \pi)^{4}} \frac{d^{4} q}{(2 \pi)^{4}} \frac{(k+q)^{2}+q^{2}-k^{2}}{k^{2}\left(1+k^{2 n} / \Lambda^{2 n}\right) l^{2}\left(1+l^{2 n} / \Lambda^{2 n}\right) q^{4}(q+k)^{4}(q+l)^{2}}= \\
& =\frac{1}{16 \pi^{2}} \int \frac{d^{4} k}{(2 \pi)^{4}} \frac{d^{4} l}{(2 \pi)^{4}} \frac{1}{\left(1+k^{2 n} / \Lambda^{2 n}\right)\left(1+l^{2 n} / \Lambda^{2 n}\right) k^{4} l^{4}},
\end{aligned}
$$

доказательство которого приведено в приложении Б. (Вновь подчеркнем, что в этом выражении из области интегрирования исключены бесконечно малые окрестности инфракрасных расходимостей.) С использованием тождества (41) и других аналогичных тождеств (см. [25]) правая часть формулы (40) может быть переписана в виде

$$
\frac{1}{4 \pi^{2}} \Lambda \frac{d}{d \Lambda} \int \frac{d^{4} k}{(2 \pi)^{4}} \frac{d^{4} l}{(2 \pi)^{4}} \frac{e^{4}}{\left(1+k^{2 n} / \Lambda^{2 n}\right)\left(1+l^{2 n} / \Lambda^{2 n}\right)}\left\{\frac{1}{2 k^{4} l^{4}}-\frac{1}{k^{4} l^{2}(k+l)^{2}}\right\} .
$$

В приложении Б этот интеграл вычислен до конца и показано, что результат является некоторой конечной постоянной. Тем не менее в целях установления связи трехпетлевой $\beta$-функции и двухпетлевой аномальной размерности более удобно записать это выражение в виде

$$
\begin{aligned}
& \frac{1}{16 \pi^{2}} \Lambda \frac{d}{d \Lambda}\left(-\int \frac{d^{4} k}{(2 \pi)^{4}} \frac{d^{4} l}{(2 \pi)^{4}} \frac{2 e^{4}}{k^{2} l^{2}(k+p)^{2}(l+p)^{2}\left(1+k^{2 n} / \Lambda^{2 n}\right)\left(1+l^{2 n} / \Lambda^{2 n}\right)}-\right. \\
& \quad-\int \frac{d^{4} k}{(2 \pi)^{4}} \frac{d^{4} l}{(2 \pi)^{4}} \frac{4 e^{4}}{k^{2} l^{2}(l+p)^{2}(k+l+p)^{2}\left(1+k^{2 n} / \Lambda^{2 n}\right)\left(1+l^{2 n} / \Lambda^{2 n}\right)}+ \\
& \left.+\int \frac{d^{4} k}{(2 \pi)^{4}} \frac{d^{4} l}{(2 \pi)^{4}} \frac{4 e^{4}(k+l+2 p)^{2}}{k^{2}(k+p)^{2} l^{2}(l+p)^{2}(k+l+p)^{2}\left(1+k^{2 n} / \Lambda^{2 n}\right)\left(1+l^{2 n} / \Lambda^{2 n}\right)}\right)\left.\right|_{p=0},
\end{aligned}
$$

поскольку такая форма записи наиболее удобна для получения тождества (25).

ПРИЛОЖЕНИЕ Б

\section{Некоторые вспомогательные тождества}

Вычисление интеграла (42) производится следуюшим образом. Вначале, учитывая симметрию относительно замены $k \leftrightarrow l$, переписываем формулу (42) в виде

$$
\begin{aligned}
\left.\Lambda \frac{d}{d \Lambda} f_{\bigcirc}\right|_{p=0}= & -\frac{1}{4 \pi^{2}} \Lambda \frac{d}{d \Lambda} \int \frac{d^{4} k}{(2 \pi)^{4}} \frac{d^{4} l}{(2 \pi)^{4}} \times \\
& \times \frac{e^{4}}{\left(1+k^{2 n} / \Lambda^{2 n}\right)\left(1+l^{2 n} / \Lambda^{2 n}\right)} \frac{k^{2}+l^{2}-(k+l)^{2}}{2 k^{4} l^{4}(k+l)^{2}}= \\
= & \frac{n}{\pi^{2}} \int \frac{d^{4} k}{(2 \pi)^{4}} \frac{d^{4} l}{(2 \pi)^{4}} \frac{e^{4} l^{2 n} / \Lambda^{2 n}}{\left(1+k^{2 n} / \Lambda^{2 n}\right)\left(1+l^{2 n} / \Lambda^{2 n}\right)^{2}} \frac{k_{\mu} l_{\mu}}{k^{4} l^{4}(k+l)^{2}} .
\end{aligned}
$$


Затем в интеграле по $d^{4} k$ переходим к четырехмерным сферическим координатам, $\left(k, \theta_{1}, \theta_{2}, \varphi\right)$, в которых мера интегрирования по угловым переменным записывается в виде

$$
d \Omega=d \theta_{1} d \theta_{2} d \varphi \sin ^{2} \theta_{1} \sin \theta_{2} .
$$

При этом четвертую ось мы будем направлять вдоль вектора $l_{\mu}$ :

$$
\begin{aligned}
\left.\Lambda \frac{d}{d \Lambda} f_{\bigcirc}\right|_{p=0}= & \frac{n}{\pi^{2}} \int \frac{d^{4} l}{(2 \pi)^{4}} \frac{1}{(2 \pi)^{4}} \int_{0}^{\infty} d k \int_{0}^{\pi} d \theta_{1} \sin ^{2} \theta_{1} \int_{0}^{\pi} d \theta_{2} \sin \theta_{2} \times \\
& \times \int_{0}^{2 \pi} d \varphi \frac{\cos \theta_{1}}{\left(1+k^{2 n} / \Lambda^{2 n}\right)\left(1+l^{2 n} / \Lambda^{2 n}\right)^{2}} \frac{e^{2 n}\left(k^{2}+2 k l \cos \theta_{1}+l^{2}\right)}{}
\end{aligned}
$$

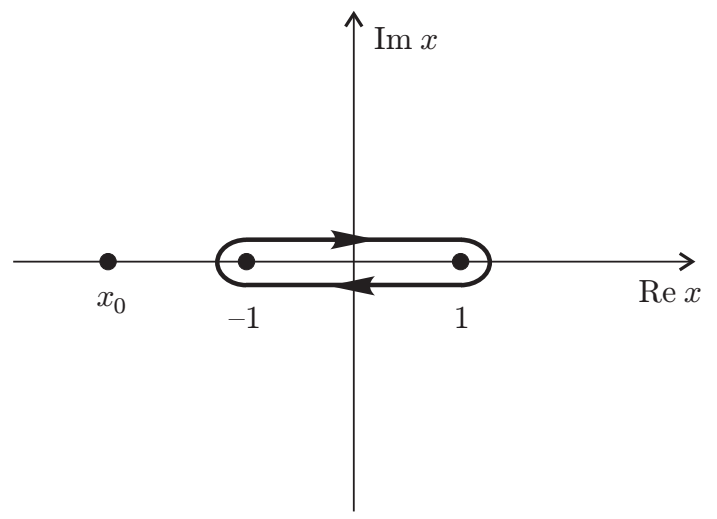

Рис. 10. Контур $C$ для вычисления интеграла по угловой переменной $x$.

Совершая замену переменных $x=\cos \theta_{1}$, можно свести интеграл по угловым переменным к интегралу по контуру $C$, показанному на рис. 10 :

$$
4 \pi \int_{-1}^{1} d x \frac{x \sqrt{1-x^{2}}}{k^{2}+2 k l x+l^{2}}=2 \pi \oint_{C} d x \frac{x \sqrt{1-x^{2}}}{k^{2}+2 k l x+l^{2}}
$$

Вычисляя этот интеграл стандартным образом по теории вычетов, получаем

$$
2 \pi \oint_{C} d x \frac{x \sqrt{1-x^{2}}}{k^{2}+2 k l x+l^{2}}=\left\{\begin{array}{cc}
-\frac{\pi^{2} l}{k^{3}}, & k \geqslant l, \\
-\frac{\pi^{2} k}{l^{3}}, & l \geqslant k .
\end{array}\right.
$$

Поэтому

$$
\begin{aligned}
\left.\Lambda \frac{d}{d \Lambda} f \bigcirc\right|_{p=0}= & -\frac{n e^{4}}{8 \pi^{2}(2 \pi)^{4}} \int_{0}^{\infty} d l \frac{l^{2 n} / \Lambda^{2 n}}{\left(1+l^{2 n} / \Lambda^{2 n}\right)^{2}} \times \\
& \times\left(\int_{l}^{\infty} d k \frac{l}{k^{3}\left(1+k^{2 n} / \Lambda^{2 n}\right)}+\int_{0}^{l} d k \frac{k}{l^{3}\left(1+k^{2 n} / \Lambda^{2 n}\right)}\right) .
\end{aligned}
$$


Совершая в первом интеграле замену переменных $x=l^{2} / \Lambda^{2}, y=\Lambda^{2} / k^{2}$, а во втором замену $x=\Lambda^{2} / l^{2}, y=k^{2} / \Lambda^{2}$, получаем

$$
\begin{aligned}
\left.\Lambda \frac{d}{d \Lambda} f \bigcirc\right|_{p=0}= & -\frac{n e^{4}}{32 \pi^{2}(2 \pi)^{4}} \int_{0}^{\infty} d x \frac{x^{n}}{\left(1+x^{n}\right)^{2}} \int_{0}^{1 / x} \frac{d y}{1+y^{-n}}- \\
& -\frac{n e^{4}}{32 \pi^{2}(2 \pi)^{4}} \int_{0}^{\infty} d x \frac{x^{n}}{\left(1+x^{n}\right)^{2}} \int_{0}^{1 / x} \frac{d y}{1+y^{n}}= \\
= & -\frac{n e^{4}}{32 \pi^{2}(2 \pi)^{4}} \int_{0}^{\infty} d x \frac{x^{n-1}}{\left(1+x^{n}\right)^{2}}=-\frac{e^{4}}{32 \pi^{2}(2 \pi)^{4}}=-\frac{\alpha^{2}}{32 \pi^{4}} .
\end{aligned}
$$

Докажем теперь тождество (41). Для этого интеграл по $d^{4} q$ в выражении

$$
I=\int \frac{d^{4} k}{(2 \pi)^{4}} \frac{d^{4} l}{(2 \pi)^{4}} \frac{d^{4} q}{(2 \pi)^{4}} \frac{2\left(q^{2}+q_{\mu} k_{\mu}\right)}{k^{2}\left(1+k^{2 n} / \Lambda^{2 n}\right) l^{2}\left(1+l^{2 n} / \Lambda^{2 n}\right) q^{4}(q+k)^{4}(q+l)^{2}}
$$

удобно записать в четырехмерных сферических координатах:

$$
\begin{aligned}
I= & \int \frac{d^{4} k}{(2 \pi)^{4}} \frac{d^{4} l}{(2 \pi)^{4}} \frac{1}{k^{2}\left(1+k^{2 n} / \Lambda^{2 n}\right) l^{2}\left(1+l^{2 n} / \Lambda^{2 n}\right)} \times \\
& \times \frac{1}{(2 \pi)^{4}} \int d \Omega \int_{0}^{\infty} d q \frac{2(q+q k \cos \alpha)}{\left(q^{2}+2 q k \cos \alpha+k^{2}\right)^{2}\left(q^{2}+2 q l \cos \beta+l^{2}\right)}= \\
= & -\int \frac{d^{4} k}{(2 \pi)^{4}} \frac{d^{4} l}{(2 \pi)^{4}} \frac{1}{k^{2}\left(1+k^{2 n} / \Lambda^{2 n}\right) l^{2}\left(1+l^{2 n} / \Lambda^{2 n}\right)} \times \\
& \times \frac{1}{(2 \pi)^{4}} \int d \Omega \int_{0}^{\infty} d q \frac{1}{\left(q^{2}+2 q l \cos \beta+l^{2}\right)} \frac{\partial}{\partial q} \frac{1}{\left(q^{2}+2 q k \cos \alpha+k^{2}\right)},
\end{aligned}
$$

где через $\alpha$ обозначен угол между четырехмерными векторами $k$ и $q$, а через $\beta$ - угол между векторами $q$ и $l$. Производя в последнем интеграле интегрирование по частям, после несложных преобразований получаем

$$
\begin{aligned}
I= & \int \frac{d^{4} k}{(2 \pi)^{4}} \frac{d^{4} l}{(2 \pi)^{4}} \frac{1}{k^{2}\left(1+k^{2 n} / \Lambda^{2 n}\right) l^{2}\left(1+l^{2 n} / \Lambda^{2 n}\right)} \times \\
& \times\left\{\frac{1}{8 \pi^{2}} \frac{1}{k^{2} l^{2}}-\int \frac{d^{4} q}{(2 \pi)^{4}} \frac{2\left(q^{2}+q_{\mu} l_{\mu}\right)}{q^{4}(q+k)^{2}(q+l)^{4}}\right\} .
\end{aligned}
$$

Последний интеграл в этом выражении с очевидностью равен $I$. Поэтому окончательно получаем

$$
I=\frac{1}{16 \pi^{2}} \int \frac{d^{4} k}{(2 \pi)^{4}} \frac{d^{4} l}{(2 \pi)^{4}} \frac{1}{\left(1+k^{2 n} / \Lambda^{2 n}\right)\left(1+l^{2 n} / \Lambda^{2 n}\right) k^{4} l^{4}} .
$$

Тем же самым способом можно получить и другие аналогичные тождества, а также ряд тождеств для диаграмм, содержаших внутренние линии полей Паули-Вилларса. 


\section{Список литературы}

[1] S. Ferrara, B. Zumino. Nucl. Phys. B. 1975. V. 87. P. 207; T. E. Clark, O. Piquet, K. Sibold. Nucl. Phys. B. 1978. V. 143. P. 445; O. Piquet, K. Sibold. Nucl. Phys. B. 1982. V. 196. P. $428 ;$ P. 447.

[2] S. L. Adler, W. A. Bardeen. Phys. Rev. 1969. V. 182. P. 1517.

[3] А. Славнов, Л. Фаддеев. Введение в квантовую теорию калибровочных полей. М.: Наука, 1988.

[4] S. L. Adler, J. C. Collins, A. Duncan. Phys. Rev. D. 1977. V. 15. P. 1712.

[5] V. A. Novikov, M. A. Shifman, A. I. Vainstein, V. I. Zakharov. Phys. Lett. B. 1985. V. 157. P. 169.

[6] P. S. Howe, K. S. Stelle, P. S. West. Phys. Lett. B. 1983. V. 124. P. 55.

[7] O. V. Tarasov, V. A. Vladimirov. Phys. Lett. B. 1980. V. 96. P. 94; M. T. Grisaru, M. Rocek, W. Siegel. Phys. Rev. Lett. 1980. V. 45. P. 1063; W. Caswell, D. Zanon. Phys. Lett. B. 1980. V. 100. P. 152.

[8] M. Shifman, A. Vainstein. Nucl. Phys. B. 1986. V. 277. P. 456.

[9] V. Novikov, M. Shifman, A. Vainstein, V. Zakharov. Phys. Lett. B. 1985. V. 166. P. 329.

[10] L. Avdeev, O. Tarasov. Phys. Lett. B. 1982. V. 112. P. 356; I. Jack, D. Jones, C. North. Nucl. Phys. B. 1996. V. 473. P. 308; Phys. Lett. B. 1996. V. 386. P. 138.

[11] I. Jack, D. R. T. Jones. Regularisation of supersymmetric theories. hep-ph/9707278.

[12] I. Jack, D. R. T. Jones, C. G. North. Nucl. Phys. B. 1997. V. 486. P. 479.

[13] D. Z. Freedman, K. Johnson, J. I. Latorre. Nucl. Phys. B. 1992. V. 371. P. 353.

[14] J. Mas, M. Perez-Victoria, C. Seijas. JHEP. 2002. V. 0203. P. 049; hep-th/0202082.

[15] N. Arkani-Hamed, H. Mirayama. JHEP. 2000. V. 0006. P. 030; hep-th/9707133.

[16] A. A. Славнов. ТМф. 1975. T. 23. № 1. C. 3; T. Bakeyev, A. Slavnov. Mod. Phys. Lett. A. 1996. V. 11. P. 1539.

[17] P. Pronin, K. Stepanyantz. Phys. Lett. B. 1997. V. 414. P. 117.

[18] A. Soloshenko, K. Stepanyantz. Two-loop renormalization of $N=1$ supersymmetric electrodynamics, regularized by higher derivatives. hep-th/0203118.

[19] А. А. Солошенко, К. В. Степаньяни. ТМФ. 2003. Т. 134. № 3. С. 430.

[20] K. В. Степаньяни. ТМФ. 2004. Т. 140. № 1. С. 53.

[21] W. Siegel. Phys. Lett. B. 1979. V. 84. P. 193.

[22] G. 't Hooft, M. Veltman. Nucl. Phys. B. 1972. V. 44. P. 189.

[23] W. Siegel. Phys. Lett. B. 1980. V. 94. P. 37.

[24] П. Уэст. Введение в суперсимметрию и супергравитацию. М.: Мир, 1989.

[25] A. Soloshenko, K. Stepanyantz. Three-loop $\beta$-function for $N=1$ supersymmetric electrodynamics, regularized by higher derivatives. hep-th/0304083.

[26] Д. И. Казаков. Письма в ЖЭТФ. 1985. Т. 41. С. 272.

[27] P. West. Nucl. Phys. B. 1986. V. 268. P. 113.

[28] A. A. Slavnov. Phys. Lett. B. 2001. V. 518. P. 195; A. А. Славнов. ТМФ. 2002. Т. 130. C. 3.

[29] А. А. Славнов, К. В. Степаньянц. ТМФ. 2003. Т. 135. С. 265.

[30] А. А. Славнов, К. В. Степаньяни. ТМФ. 2004. Т. 139. № 2. С. 179.

Поступила в редакцию 22.X.2003 г., после доработки 12.I.2004 г. 